\title{
7th International Workshop on Web Information Systems Modeling (WISM 2010)
}

\section{Preface}

The international workshop on Web Information Systems Modeling (WISM) aims to study the latest developments in modeling of Web Information Systems (WIS). This is the seventh edition of this workshop which follows successful editions organized in Amsterdam (2009), Barcelona (2008), Trondheim (2007), Luxembourg (2006), Sydney (2005), and Riga (2004).

In the past, storyboards have been successfully used for modeling WIS. As there is an increasing need to support complex interactions between users and WIS, in the first paper by Berg et al. the authors propose extensions to storyboards based on speech dialogues. The common dialogue forms are supported by dialogue patterns.

Currently there are various formats for Web logs which makes log analysis a difficult process. The second paper by Hernandez et al. proposes a unified model for Web log data that is subsequently used for deriving a multidimensional model. The data transformations are specified using Query/View/Transformation rules and are used in a fully automatic process.

There are many Web applications that have a REpresentational State Transfer (REST) architecture and XML-based data storage. As REST is stateless, it is difficult to support auditing and accountability for these applications. The third paper by Graf et al. proposes an opportunistic locking mechanism that ensures easy modifications of the allocation of XML nodes and scalable integrity verification based on the XML tree structure.

A lot of the current research is done by means of collaborations between individuals that often belong to different research groups. Finding the right partner and analysing collaborations are complex tasks. The fourth paper by Lopes et al. proposes a framework for recommending collaborations in the context of Web social networks. The authors present the architecture of the framework, the metrics involved in recommending collaborations, and preliminary evaluation results.

The sentiment emerging from Web documents is an influential factor for business decisions. Unlike other approaches that use the bag-of-words model to compute sentiment scores for documents, the last paper by Hogenboom et al. proposes to consider the document narrative structure for determining the document sentiment. Depending on the position in the document rhetorical structure, text fragments can have different impacts during sentiment computations.

Based on this selection of papers and topics relevant to the workshop goals, we invite the interested reader to have a closer look at the articles gathered in the proceedings. We would like to thank all the authors, reviewers, and participants for their contributions and support, making the organization of this new edition of the workshop possible.

July 2010

Flavius Frasincar Geert-Jan Houben Philippe Thiran 